\title{
Vingt ans de transitions agricoles et rurales à l'Est. Quels enseignements?
}

\section{Alain Pouliquen}

\section{(2) OpenEdition}

1 Journals

\section{Édition électronique}

URL : http://journals.openedition.org/economierurale/3212

DOI : $10.4000 /$ economierurale.3212

ISSN : 2105-2581

\section{Éditeur}

Société Française d'Économie Rurale (SFER)

\section{Édition imprimée}

Date de publication : 21 octobre 2011

Pagination : 5-9

ISSN : 0013-0559

\section{Référence électronique}

Alain Pouliquen, « Vingt ans de transitions agricoles et rurales à l'Est. Quels enseignements ? », Économie rurale [En ligne], 325-326 | septembre-décembre 2011, mis en ligne le 17 octobre 2013, consulté le 30 avril 2019. URL : http://journals.openedition.org/economierurale/3212 ; DOI : 10.4000/ economierurale.3212 


\title{
Vingt ans de transitions agricoles et rurales à l'Est Quels enseignements?
}

\author{
Alain POULIQUEN \\ Directeur de recherche INRA
}

V ingt ans après la rupture systémique de 1990 et six ans après le premier élargissement de l'UE à l'Est, cette livraison d'Économie rurale a mobilisé un réseau interdisciplinaire de chercheurs bien insérés dans le débat scientifique international. S'appuyant sur l'observation de terrains très divers, ces douze articles traitent de questions dont les enjeux scientifiques, économiques et sociaux dépassent largement les particularités du champ étudié.

En effet, ils confirment que les dynamiques observées ont largement déjoué les préconceptions et prévisions dominantes des «transitions » postcommunistes, imposé un profond renouvellement de leurs cadres théoriques et méthodologiques, et interpellent les politiques agricoles à l'œuvre, dont la politique agricole commune. À cet égard l'introduction schématique qui suit aux points-clés de chacun des articles montre que, au-delà de leur grande diversité d'objets et d'approches, une certaine cohérence d'ensemble transparait de leurs convergences et complémentarités. Pour en faciliter la lecture, nous commencerons par les articles de portée plus ou moins synthétique, avant d'introduire les analyses par pays et/ou ciblées sur une région, un village, un sous-secteur ou une institution.

\section{Les paysages recomposés des nouveaux États membres}

\section{Les évolutions de l'agriculture}

Neuf des douze articles traitent, principalement ou exclusivement, des Nouveaux États membres (NEM) de l'UE. Vu la grande diversité de leurs poids respectifs et de leurs structures, un cadrage économique, calé sur les statistiques européennes, s'imposait pour cet ensemble. C'est l'objet de l'article de Gilles Bazin et Lise Bourdeau-Lepage, qui proposent une analyse des évolutions fonctionnelles et structurelles des agricultures des NEM dans les années 2000-2008, soit jusqu'au déclenchement de l'actuelle crise financière-économique ${ }^{1}$.

Impulsés par la poursuite d'une forte croissance globale et par l'accès aux aides de la Pac, la reprise agricole et les gains de productivité ont globalement évité le bouleversement, a priori très redouté de part et d'autre, des équilibres Est-Ouest des productions et échanges agricoles mutuels. Mais les écarts de productivité restent substantiels et l'extrême dualisme structurel hérité de l'ère communiste reste marqué. D'un côté en effet, les grandes et très grandes exploitations sociétaires et individuelles, issues de l'ancien secteur socialisé, sont très renforcées par les aides communautaires, de l'autre une partie notable des minifundia de subsistance a trouvé des voies multifonctionnelles de résistance. Toutefois, dans la plupart des NEM, les exploitations «moyennes » ont conquis ou maintenu (notamment en Pologne) une place significative, mais le plus souvent sans accéder au modèle « professionnel » ouest européen.

1. Élaborée parallèlement et sans concertation mutuelle, mon étude parue en octobre 2010 dans le Demeter 2011 converge en large part avec cette analyse. 


\section{INTRODUCTION}

\section{Les réformes foncières}

Céline Bignebat et Laure Latruffe examinent les multiples obstacles ou freins que les conditions sociales, politiques et administratives de la privatisation des terres ont opposé au libre jeu théorique de leur réallocation optimale par la concurrence sur des marchés fonciers dont la formation est restée très imparfaite. Tout ceci a étroitement limité les transactions sur la propriété, alors que les marchés de location ont été nettement plus actifs, mais souvent sur le mode informel. La propriété foncière issue des restitutions est généralement restée hautement fragmentaire et indivise. Les auteures montrent comment cette configuration a déterminé, en large part, la prédominance recomposée du dualisme foncier hérité de l'ère communiste, et la faible émergence d'exploitations familiales moyennes de profil ouest européen.

\section{La ruralité recomposée}

Guillaume Lacquement, Marie-Claude Maurel et Jean-Claude Raynal explorent les trajectoires d'une ruralité recomposée en Europe centrale. La question est cruciale pour l'agriculture elle-même, car la rupture systémique de 1990 a aboli les relations organiques qui lui asservissaient antérieurement les activités rurales, sans relai adéquat de nouveaux pouvoirs et financements territoriaux. Mobilisant une typologie des itinéraires de développement rural, l'étude met en évidence ses fortes et croissantes inégalités territoriales et leurs facteurs explicatifs, dont la politique communautaire de développement rural et les structures économiques régionales héritées, ainsi que leurs implications démographiques et socioéconomiques.

\section{L'évolution des échanges agricoles}

Utilisant les indicateurs ad hoc, l'analyse statistique, de Marie-Line Duboz et Julie Le Gallo, des échanges agricoles entre l'UE à 15 et les PECO de la première vague d'adhésions montre une spécialisation croissante des seconds dans les productions et exportations de lait, viandes et céréales, qui les met en concurrence avec les agricultures du Nord de l'ex-UE à 15.

\section{Les regards et les nuances par pays}

\section{Pologne et Roumanie face à la Pac}

Catherine Darrot et Béatrice Von Hirschhausen interrogent le rôle de la Pac dans la «néo-dualisation» structurelle susmentionnée, dans les deux cas importants de la Pologne et de la Roumanie. Les auteures mettent en évidence un décalage radical et croissant entre, d'une part le projet de la Pac plaquant à l'Est la voie de modernisation historiquement prédominante dans l'UE15 et d'autre part, la réalité des modèles paysans polonais et roumain et de leurs trajectoires endogènes de résistance. Ceci ressort d'une analyse sémantique des objectifs officiels des plans de développement rural et de leur évolution, et du constat chiffré de la contradiction flagrante entre l'objectif de réduction des disparités régionales, et la répartition effective des aides directes et structurelles de la Pac, par région et type d'exploitation.

\section{L'Allemagne de l'Est face à la décollectivisation}

Le « retour vers le futur » de Michel Streith sur les premières années postsocialistes de l'agriculture est-allemande, remet en lumière l'impact du coup de force politicoéconomique initial qu'a été, pour la population agricole et rurale concernée, une 
décollectivisation conduisant immédiatement aux licenciements massifs. Ceci a résulté conjointement de l'immersion immédiate des exploitations collectives dans le marché communautaire sans leurs subventions antérieures, et de l'absence de politique adéquate de soutien à l'installation familiale sur les terres restituées. Ainsi un marché de dupes (restitution des terres contre perte d'emploi) inversait le pacte communiste imposé (collectivisation contre création de nombreux emplois agricoles et ruraux). Soulignons que, précipité par le contexte particulier de la réunification allemande, ce même basculement a aussi affecté, à divers rythmes et degrés, toutes les autres agricultures esteuropéennes.

\section{Quelle adéquation pour la Hongrie?}

Perrine Vandenbroucke et Istvàn Fehér traitent de la dynamique des structures agraires en Hongrie. L'étude s'appuie principalement sur une enquête dans une région proche de Budapest, et des entretiens complémentaires dans d'autres régions. Elle illustre la tendance générale susmentionnée à une néo-dualisation structurelle de l'agriculture, et en élucide finement les orientations et les mécanismes à l'échelle microéconomique. Le poids de l'héritage structurel n'est pas seulement inertiel, car ses recompositions apparaissent très actives et inventives, sous la pression conjointe des marchés, du chômage rural, des opportunités et contraintes des marchés fonciers, du développement local et des soutiens et normes de la Pac. Pour les auteurs, l'échec du «placage» du modèle ouest européen d'exploitation familiale moyenne questionne son adéquation future à la réalité de la plupart des NEM, voire de l'ensemble de l'UE.

\section{Un village roumain face à la propriété privée}

La monographie villageoise de Florina Gaborean apporte un éclairage anthropologique à l'explication de la trajectoire néo-dualiste des structures agraires. Citons le résumé: « ... Les pratiques coutumières de transmission patrimoniale, le rapport à la terre et le retour aux anciennes formes d'exploitation agricole ont directement influencé le processus de décollectivisation ». En fait l'article porte plus largement sur le jeu complexe des carences institutionnelles et des contraintes économiques qui explique d'un côté, ce « retour paysan au passé » et sa consolidation partielle, et de l'autre sa vulnérabilité et les débuts de son dépassement difficile, au profit d'un pôle entrepreneurial concentré. Les millions de micro-parcelles restituées restent très souvent en indivision entre héritiers, ce qui bloque leurs achats-ventes et entretient les solidarités ou les conflits intrafamiliaux.

Dans cette configuration une partie des micro-exploitations de subsistance parvient à consolider sa résistance multifonctionnelle, ce que traduit la forte montée des prix des parcelles « intravilan », vouées à l'autoconsommation. En revanche la modernisation de la production marchande sur les terres « extravilan » reste généralement problématique sur le mode familial, faute de financement. Ceci conduit au très faible prix de ces terres et à leur part élevée de friches, car les carences institutionnelles du marché foncier dissuadent leur reprise par des investisseurs extérieurs. Cette reprise semble toutefois s'amorcer par location, au profit du pôle concentré de l'agriculture. Rappelons que celuici est très majoritairement situé dans certaines autres régions, où prédominent les macro-exploitations sociétaires et individuelles issues des fermes d'État. 


\section{La marginalisation des minifundia roumaines}

Valérie Villemin, Wladimir Andreff et Étienne Montaigne illustrent le processus de marginalisation des minifundia roumaines dans le cas de la viticulture. Leur substitution de robustes cépages hybrides aux délicats cépages nobles répond rationnellement à leur manque de capital et à leur orientation majeure vers l'autoconsommation. Mais, au nom de la qualité, ceci inspire une politique communautaire et nationale d'arrachage des hybrides. De plus la fragmentation extrême des parcelles à conduit, pour réduire les coûts administratifs de distribution des aides communautaires, à des minima de surface éligible qui en excluent l'essentiel du vignoble paysan, soit $60 \%$ du vignoble national. Les auteurs soulignent les grandes pertes potentielles d'opportunités que risque d'entraîner cette politique pour le développement rural et la rénovation de la vitiviniculture nationale. Une politique alternative devrait s'appuyer sur une meilleure représentation professionnelle des minifundistes et leur médiation collective dans la gestion des aides publiques.

\section{Les Balkans et le concept des $I G$}

Magali Estève, Marguerite Paus, Dominique Barjolle et Pascal Bernardoni explorent les perspectives et les limites de filières sous Indications géographiques (IG) dans les Balkans. La diffusion du concept européen de protection des IG passe par la négociation de projets de coopération entre les acteurs économiques (producteurs, revendeurs, transformateurs) et institutionnels (experts, administrations, ONG, associations de producteurs) concernés. Les deux études de cas, en Bosnie et en Serbie, montrent que le potentiel de protection des IG, en termes de développement rural endogène, est restreint par une transition institutionnelle inachevée.

\section{La réforme agraire dans la campagne russe}

À partir de données globales et d'enquêtes auprès des acteurs dans quatre oblasts représentatifs, Estelle Lézéan analyse la trajectoire de l'agriculture russe depuis 1991, en relation avec les réformes et les politiques suivies. Dans les années 1990 la création de fermes familiales de type occidental se heurte à la résistance conjointe des appareils administratifs locaux et des travailleurs des kolkhozes et sovkhozes (KS), et leur contribution productive reste marginale. Quant à l'attribution formelle de titres de propriété à ces travailleurs, elle ne leur permet pas de s'établir séparément, notamment du fait de leur dépendance multiforme du KS. La fin des subventions antérieures et la désorganisation des circuits agro-industriels privatisés entraînent un recul catastrophique de la production collective des KS, qui contraste avec la résistance de celle des lopins de subsistance de leurs employés et l'essor du « jardinage urbain » (voir l'article suivant).

Parallèlement, la concentration graduelle des parts sociales des ex-KS entre les mains de leurs dirigeants les transforme en latifundia d'un capitalisme très dépendant des autorités locales. Suit, dans les années 2000, leur rachat sélectif par de grands investisseurs privés qui les regroupent en immenses «agro-holdings » à caractère agro-industriel, toujours sous le contrôle multiforme d'un appareil d'État désormais re-centralisé. Tout ceci tend à détruire l'ancien lien « symbiotique » des lopins ruraux avec le KS, condition de leur survie. De plus, dans un deuxième temps, les grands investisseurs privés, échaudés par les aléas des prix et des rendements agricoles, tendent à se dégager des agro-holdings. On comprend donc que la recherche d'une autonomie alimentaire nationale accrue suscite un tournant récent vers la relance des «fermes » familiales. 


\section{Le jardinage urbain de Russie : une nouvelle société}

Louiza Boukharaeva et Marcel Marloie complètent le tableau agricole russe par l'examen approfondi d'une de ses composantes majeures, souvent négligée : le jardinage urbain à grande échelle de Russie. En réponse à l'effondrement productif des ex-KS et à celui du pouvoir d'achat dans les années 1990, sa production autoconsommée a fortement cru. En 2000, ce secteur, occupant à temps partiel plus de la moitié de la population urbaine, fournissait environ $60 \%$ de son ravitaillement, et $30 \%$ de toute l'alimentation nationale. L'analyse historique des conditions institutionnelles et sociologiques de son développement montre qu' «il ne s'agit pas de la réminiscence du passé rural, ni d'un phénomène éphémère spécifique à la période soviétique », et qu'il « s'apparente à la construction d'une société nouvelle», dont les enseignements ont une portée universelle.

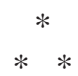

L'élargissement de l'UE à dix pays d'Europe centrale et orientale y a profondément transformé les conditions sociales, économiques et politiques du développement agricole et rural. Il convenait d'en tirer un premier bilan s'appuyant sur l'analyse des restructurations agricoles et rurales intervenues, plus largement, en « Europe de l'Est » au cours de ces vingt dernières années.

Économie rurale a offert ses colonnes à tous ces auteurs issus du monde de la recherche. À travers leurs travaux, ils répondent de manière circonstanciée aux questions que nos lecteurs peuvent légitimement se poser : depuis 1990, quels enseignements peut-on tirer de la transition postcommuniste des pays de l'Est tant au niveau global qu'au niveau des États ? Comment ces sociétés agricoles et rurales ont-elles fait face à de nouvelles contraintes et opportunités économiques ? Il en résulte un jugement général qui est celui du profond renouvellement des cadres structurels, qui interpelle les politiques agricoles à l'œuvre, dont la politique agricole commune. 